\title{
Single-incision-two port laparoscopic tubal ligation: A cost comparison and technique description
}

\author{
Nicel Taşdemir, Remzi Abal, Cem Çelik, Erson Aksu, Didem Akkuş \\ Department of Obstetrics and Gynecology, Namik Kemal University Faculty of Medicine, Tekirdağ, Turkey
}

\section{Abstract}

Objective: Laparoscopic surgery is the principal minimally invasive technique that is used for the treatment of gynecologic pathologies. The single-incision laparoscopic surgery (SILS) is another innovation in minimally invasive medicine. The cost of the procedure correlates with the fundamental materials used to access the abdominal cavity and utilize trocars.

Material and Methods: We applied the single-incision tubal ligation procedure to three patients. A 15-20-mm vertical incision was made in the umbilicus. Two trocars were inserted through the same incision at different fascial regions after insufflation of the abdomen. A 5-mm bipolar cautery was introduced through the accessory trocar, and the mid-portion of the tubes was coagulated and cut bilaterally.

Results: The postoperative periods of the three patients were uneventful. All patients were discharged on the day of surgery. No major or minor complications occurred.

Conclusion: The cost for the abdominal access will drop about $82 \%$. When we consider the low pricing for the tubal ligation procedure, the single-incision technique will be more applicable by this method. Moreover, patients will have the advantages of single-incision laparoscopic surgery with low cost. (J Turk Ger Gynecol Assoc 2015; 16: 30-1)

Keywords: Cost, laparoscopy, single incision

Received: 04 July, 2014

Accepted: 18 December, 2014

\section{Introduction}

The trend in gynecologic surgery is to be minimally invasive. Laparoscopic surgery is the principal minimally invasive technique that is used for the treatment of gynecologic pathologies. The benefits of laparoscopic surgery are less postoperative pain morbidity, shorter hospitalization duration, and shorter recovery time. The single-incision laparoscopic surgery (SILS) is another innovation in minimally invasive medicine. It was first described in 1976 by Wheeless (1) for the tubal ligation procedure and evolved with improvements in technology.

There are several techniques for female sterilization. Surgical sterilization is an effective, permanent, and safe one. Surgical sterilization could be carried out by laparoscopy, mini-laparotomy, or hysteroscopy. Laparoscopy is a widely used technique with a shorter recovery period and ease of application. Various devices are available for the execution of SILS on the market. The cost of the procedure correlates with the fundamental materials used to access the abdominal cavity and utilize trocars.

We wanted to describe an easy-to-apply and low-budget technique to execute relatively simple procedures, like tubal ligation.

\section{Material and Methods}

Three patients with the desire of permanent sterilization applied to the gynecology clinic in Namik Kemal University School of Medicine. The informed consents were obtained prior to procedures.

We applied a similar technique that was described by Podolsky et al. (2) for cholecystectomy. A single 15-20-mm vertical incision was made in the umbilicus. The Veres needle was used to maintain pneumoperitoneum at 15 $\mathrm{mm} \mathrm{Hg}$ pressure. After insufflation, a 5-mm trocar was introduced into the abdomen. A 5-mm, 30-degree scope was used to confirm intraperitoneal entrance. Afterwards, another 5-mm trocar was introduced under direct observation. In other words, two trocars were inserted through the same incision at different fascial regions (Figure 1). We used a rubin cannula to elevate the uterus after maintenance of the Trendelenburg position. Then, a 5-mm bipolar cautery was introduced through the accessory trocar, and the midportion of the tubes was coagulated and cut bilaterally. After removal of the trocars, fascial apertures were sutured with 0 Vicryl (Ethicon, Istanbul, Turkey) suture. The skin was closed with separated 3-0 Rapid Vicyl (Ethicon, Istanbul, Turkey) sutures. 


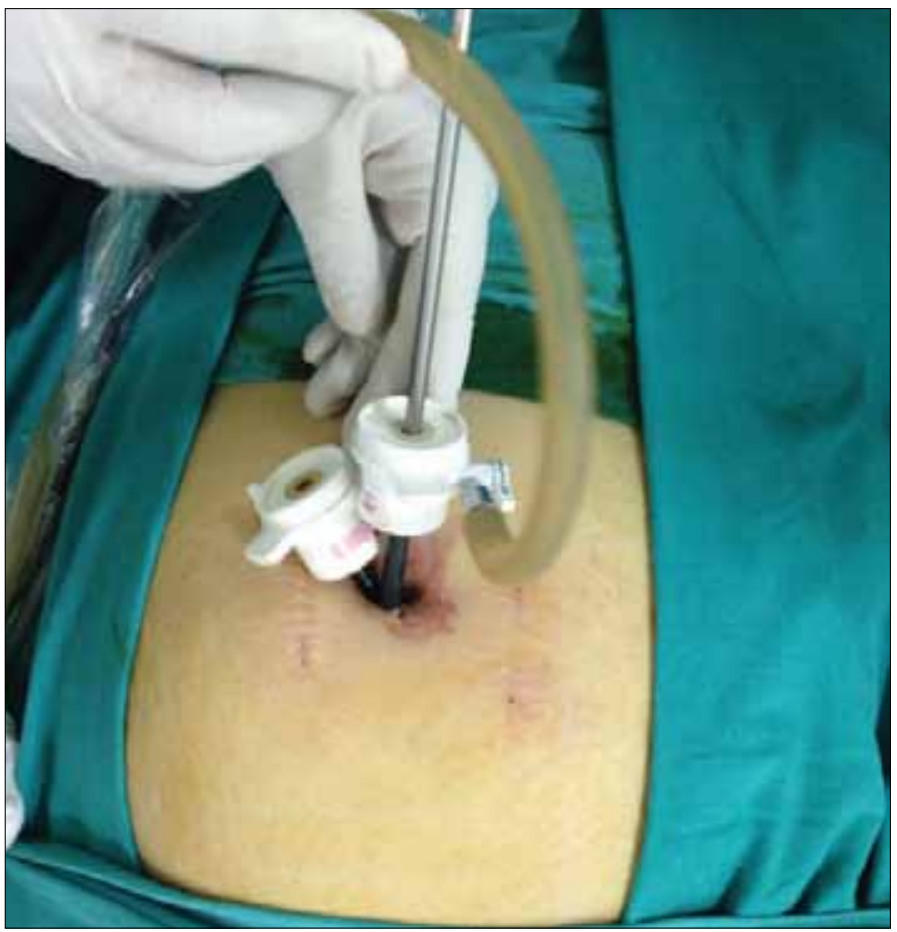

Figure 1. The view of the umbilical 5-mm trocars after insufflation of the abdomen

\section{Results}

The postoperative periods of the three patients were uneventful (Figure 2). All patients were discharged on the day of surgery. No major or minor complications occurred. The cost of abdominal access with the SILS Port (Covidien, Istanbul, Turkey) is 1058 Turkish liras (365 euros). However, the cost of the abdominal access was 192 Turkish liras (66 euros) with the technique described.

\section{Discussion}

The options for tubal ligation are mini-laparotomy, conventional laparoscopy, and single-incision laparoscopy. The laparoscopic technique is the least invasive procedure for tubal ligation. When we compared laparotomy and laparoscopy, the laparoscopy technique has advantages, such as quick convalescence, better cosmetic results, and less pain after surgery. Thus, it is the technique of choice where available. However, laparotomy is carried out under local anesthesia with mild sedation, whereas laparoscopy mostly requires general anesthesia. Usually, two or three incisions are made for the laparoscopic procedure. On the other hand, single-port access has advantages, such as better cosmetic results, less pain, reduced complications related to trocar entry, and easier closure of the fascia with a larger incision (3). Single-port laparoscopy requires instruments that are used to maintain abdominal access. The cost of the instruments is the major limitation of single-incision laparoscopy. We use the SILS Port (Covidien, Istanbul, Turkey) for single-port laparoscopic surgery. The cost of the SILS Port was 1058 Turkish liras (365 euros). However, if we use two separate 5 -mm trocars

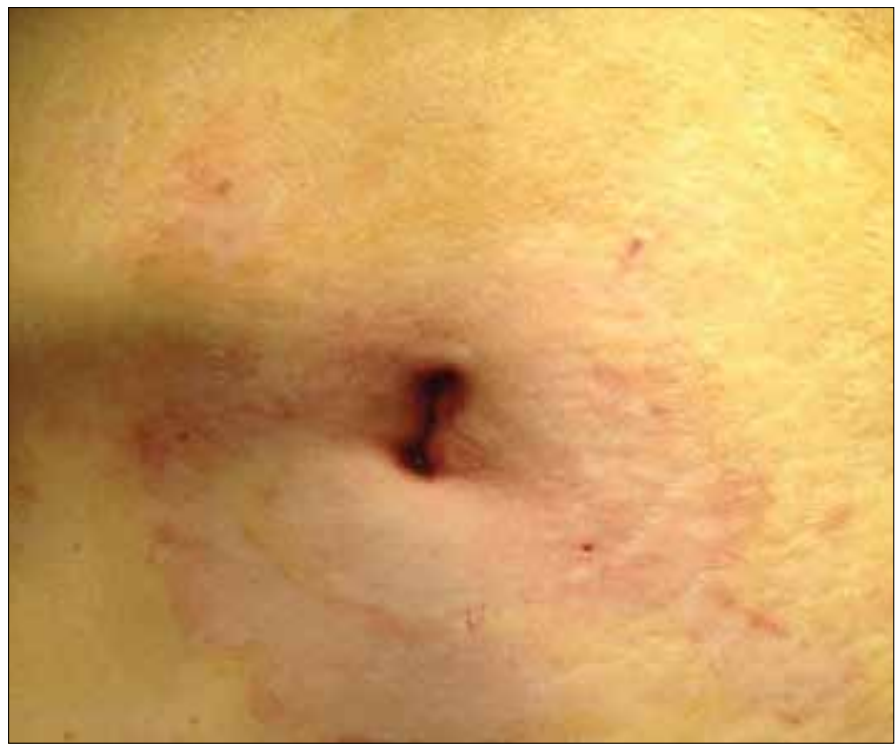

Figure 2. Early postoperative appearance of $20-\mathrm{mm}$ intraumbilical incision after closure

instead of the SILS Port, as described by Podolsky et al. (2), the cost will be 192 Turkish liras (66 euros). The cost for abdominal access will drop about $82 \%$.

Podolsky et al. (2) reported a learning curve, including 5 cases, for the three-port cholecystectomy procedure with the technique. We believe that it is not needed for the tubal ligation procedure, and a laparoscopic surgeon who is familiar with basic laparoscopy and single-port laparoscopy techniques can easily apply the technique.

The cost and effectiveness of a medical application are some of the most important aspects, and they determine the applicability of a technique. When we consider the low pricing for the tubal ligation procedure, the single-incision technique will be more applicable by this method. Moreover, patients will have the advantages of single-incision laparoscopic surgery with low cost.

Ethics Committee Approval: N/A.

Informed Consent: Written informed consent was obtained from patients who participated in this study.

Peer-review: Externally peer-reviewed.

Author contributions: Concept - N.T., R.A., C.Ç.; Design - N.T.; Supervision - N.T., Resource - N.T.; Materials - N.T., R.A., C.Ç.; Data Collection\&/or Processing - N.T., E.A., D.A.; Analysis\&/or Interpretation - N.T., E.A., D.A.; Literature Search - E.A, D.A.; Writing - N.T., R.A., C.Ç.; Critical Reviews - N.T.

Conflict of Interest: No conflict of interest was declared by the authors.

Financial Disclosure: The authors declared that this study has received no financial support.

\section{References}

1. Wheeless CR, Jr. Elimination of second incision in laparoscopic sterilization. Obstet Gynecol 1972; 39: 134-6.

2. Podolsky ER, Rottman SJ, Poblete H, King SA, Curcillo PG. Single port access (SPA) cholecystectomy: a completely transumbilical approach. J Laparoendosc Adv Surg Tech A 2009; 19: 219-22. [CrossRef]

3. Jackson T, Einarsson J. Single-port gynecologic surgery. Rev Obstet Gynecol 2010; 3: 133-9. 